\title{
STUDY OF MICROALBUMINURIA IN TYPE 2 DIABETES MELLITUS
}

\author{
Thirupathi Rao J1, Ambapurkar Mukund Madhukar ${ }^{2}$
}

${ }^{1}$ Assistant Professor, Department of General Medicine, GMC, Nizamabad.

${ }^{2}$ Assistant Professor, Department of General Medicine, GMC, Nizamabad.

\section{ABSTRACT}

\section{BACKGROUND}

Diabetes Mellitus (DM) refers to a group of common metabolic disorders that share the phenotype of hyperglycaemia. Several distinct types of DM are caused by a complex interaction of genetics and environmental factors. Microalbuminuria is defined as 30 $299 \mathrm{mg} / \mathrm{d}$ in a 24-h collection or $30-299 \mathrm{~g} / \mathrm{mg}$ creatinine in a spot collection. Microalbuminuria is a risk factor for cardiovascular disease. Once microalbuminuria is present, there is a steady decline in GFR and 50\% of individuals reach ESRD in 7 - 10 years. Once microalbuminuria develops, blood pressure rises slightly and the pathologic changes are likely irreversible.

Aim and Objectives -

1. To study the clinical profile of Type 2 Diabetes Mellitus patients with early nephropathy, i.e. Microalbuminuria.

2. To identify any specific risk factors with microalbuminuria.

\section{MATERIALS AND METHODS}

A clinical study consisting of 100 Type 2 DM patients is undertaken to investigate the pattern and magnitude of microalbuminuria and its relationship with microvascular and macrovascular complications of Type 2 Diabetes Mellitus. Study group was divided into two groups Group A: Type DM patients who were positive for microalbuminuria (Micral Positive) 50 patients, Group B: Type 2 DM patients who were negative for microalbuminuria (Micral positive) 50 patients.

\section{RESULTS}

Smoking ( $\mathrm{P}=0.016)$ observed in $32 \%$ of Micral positive patients, $12 \%$ of Micral negative patients. The present study reveals a strong association of microalbuminuria with poor glycaemic control. This study showed higher prevalence of hypertension (54\%) in Micral positive group compared to 14\% in Micral negative group. Retinopathy was seen in 44\% of Micral positive cases and 8\% of Micral negative type 2 DM. The prevalence of ischaemic heart disease was also higher in Micral positive group (28\%) compared to Micral negative group (6\%).

\section{CONCLUSION}

Age and Sex do not seem to be directly related to the development of microalbuminuria in type 2 DM patients. Smoking has a strong association with the development of microalbuminuria. Hence, discontinuation of smoking will be an important intervention in arresting the progression of early nephropathy. Poor glycaemic control is also strongly associated with type $2 \mathrm{DM}$ patients with microalbuminuria. The DCCT study shows the importance of tight glycaemic control in the prevention of microvascular complications. So a good glycaemic control should be aimed at in patients with early nephropathy. Hypertension has a strong association with the development of microalbuminuria. Ischaemic Heart Disease is also common in type 2 DM with microalbuminuria. Retinopathy has a very strong association with microalbuminuria. Neuropathy another microvascular complication is also strongly associated with microalbuminuria. Serum cholesterol, Triglycerides and LDL levels were significantly higher in microalbuminuric patients.

\section{KEYWORDS}

Diabetes Mellitus, Microalbuminuria.

HOW TO CITE THIS ARTICLE: Rao TJ, Madhukar AM. Study of microalbuminuria in type 2 diabetes mellitus. J. Evolution Med. Dent. Sci. 2017;6(14):1110-1114, DOI: 10.14260/Jemds/2017/241

\section{BACKGROUND}

Diabetes Mellitus (DM) refers to a group of common metabolic disorders that share the phenotype of hyperglycaemia. Several distinct types of DM are caused by a complex interaction of genetics and environmental factors.

Financial or Other, Competing Interest: None.

Submission 09-01-2017, Peer Review 02-02-2017,

Acceptance 08-02-2017, Published 16-02-2017.

Corresponding Author:

Dr. Thirupathi Rao J,

Assistant Professor,

Department of General Medicine,

GMC, Nizamabad.

E-mail: drthirujalagam1234@gmail.com

DOI: $10.14260 /$ jemds $/ 2017 / 241$
Depending on the aetiology of the DM, factors contributing to hyperglycaemia include reduced insulin secretion, decreased glucose utilisation and increased glucose production. The metabolic dysregulation associated with DM causes secondary pathophysiological changes in multiple organ systems that impose a tremendous burden on the individual with diabetes and on the healthcare system.

DM is classified on the basis of the pathogenic process that leads to hyperglycaemia as opposed to earlier criteria, such as age of onset or type of therapy. The two broad categories of DM are designated type 1 and type 2 . Both types of diabetes are preceded by a phase of abnormal glucose homeostasis as the pathogenic processes progress. Type 1 DM is the result of complete or near-total insulin deficiency. 
Type $2 \mathrm{DM}$ is a heterogeneous group of disorders characterised by variable degrees of insulin resistance, impaired insulin secretion and increased glucose production. Distinct genetic and metabolic defects in insulin action and/or secretion give rise to the common phenotype of hyperglycaemia in type $2 \mathrm{DM}$.

The chronic complications of DM affect many organ systems and are responsible for the majority of morbidity and mortality associated with the disease. Chronic complications can be divided into vascular and nonvascular complications. The vascular complications of DM are further subdivided into microvascular (retinopathy, neuropathy, nephropathy) and macrovascular complications [Coronary Heart Disease (CHD), Peripheral Arterial Disease (PAD), cerebrovascular disease]. The microvascular complications of both type 1 and type 2 DM result from chronic hyperglycaemia.

One theory is that increased intracellular glucose leads to the formation of advanced glycosylation end products (AGEs), which bind to a cell surface receptor via the non-enzymatic glycosylation of intra- and extra-cellular proteins.

Diabetic nephropathy is the leading cause of End Stage Renal Disease (ESRD) worldwide and a leading cause of DMrelated morbidity and mortality. Both microalbuminuria and macroalbuminuria in individuals with DM are associated with increased risk of cardiovascular disease. Individuals with diabetic nephropathy commonly have diabetic retinopathy.

The mechanisms by which chronic hyperglycaemia leads to ESRD, though incompletely defined, involve the effects of soluble factors (growth factors, angiotensin II, endothelin, AGEs), haemodynamic alterations in the renal microcirculation (glomerular hyperfiltration or hyperperfusion, increased glomerular capillary pressure), and structural changes in the glomerulus (increased extracellular matrix, basement membrane thickening, mesangial expansion and fibrosis). Some of these effects may be mediated through angiotensin II receptors. Smoking accelerates the decline in renal function.

Microalbuminuria is defined as $30-299 \mathrm{mg} / \mathrm{d}$ in a 24-h collection or $30-299 \mathrm{~g} / \mathrm{mg}$ creatinine in a spot collection. Microalbuminuria is a risk factor for cardiovascular disease. Once macroalbuminuria is present, there is a steady decline in GFR and $50 \%$ of individuals reach ESRD in 7 - 10 years. Once macroalbuminuria develops, blood pressure rises slightly and the pathologic changes are likely irreversible.

The nephropathy that develops in type 2 DM differs from that of type $1 \mathrm{DM}$ in the following respects: (1) Microalbuminuria or macroalbuminuria may be present when type $2 \mathrm{DM}$ is diagnosed reflecting its long asymptomatic period; (2) Hypertension more commonly accompanies microalbuminuria or macroalbuminuria in type $2 \mathrm{DM}$; and (3) Microalbuminuria may be less predictive of diabetic nephropathy and progression to macroalbuminuria in type 2 DM. Finally, it should be noted that albuminuria in type 2 DM may be secondary to factors unrelated to DM such as hypertension, Congestive Heart Failure (CHF), prostate disease or infection. ${ }^{1}$

Diabetic nephropathy is the most common cause of End Stage Renal Disease (ESRD). Type 2 DM patients account for $40-90 \%$ of this burden in the U.S. In India too diabetic Nephropathy is a major cause of ESRD with a prevalence of $26 \%$ as reported by Chugh K. S. et al. ${ }^{2}$
From Chandigarh and $26.7 \%$ as reported by Mani M. K. et $\mathrm{al}^{3}$ from Madras. Also in Dr. Mani's series of cases, Diabetic Nephropathy was found in $18.7 \%$ of all cases of nephrotic syndrome.

\section{Aim and Objectives}

- To study the clinical profile of Type 2 Diabetes Mellitus patients with early nephropathy, i.e. Microalbuminuria.

- To identify any specific risk factors with microalbuminuria.

\section{MATERIALS AND METHODS \\ Study Design}

A clinical study consisting of 100 Type 2 DM patients is undertaken to investigate the pattern and magnitude of microalbuminuria and its relationship with microvascular and macrovascular complications of Type $2 \mathrm{DM}$.

In this study, 380 consecutive cases screened during the year 2009 - 2011. A detailed history, clinical examination and necessary laboratory tests were carried out. From these 280 patients having overt proteinuria, UTI, CCF, moderate-tosevere hypertension and pregnancy were excluded.

100 patients were screened using the Micral strip for assessing as per the procedure described earlier. Based on the results, the patients were divided into two groups.

One hundred patients of diabetes (type II DM) admitted to MNR Hospital attached to the MNR Medical College were studied. The patients were taken from the medical wards of the hospital based on random selection. Patients were considered to be diabetic based on WHO (2) criteria for diagnosis of diabetes mellitus.

\section{Which is -}

1. Symptoms of diabetes mellitus plus a random glucose concentration > $200(11.1 \mathrm{mmol} / \mathrm{l})$. The classic symptoms of diabetes mellitus include polyuria, polydipsia and unexplained weight loss.

\section{OR}

2. Fasting blood glucose $>126 \mathrm{mg} / \mathrm{dL}(7.0 \mathrm{mmol} / \mathrm{L})$. Fasting is defined as no caloric intake for at least 8 hours.

\section{OR}

3. 2-hour post prandial glucose $>200 \mathrm{mg} / \mathrm{dL}(11.1$ $\mathrm{mmol} / \mathrm{L})$. Among diabetics, the above criteria were considered to include the patients for the study.

This clinical study consisting of hundred Type 2 DM patients is undertaken to investigate the pattern and magnitude of microalbuminuria and its relationship with microvascular and macrovascular complications of Type 2 DM. All patients having overt macroalbuminuria detected by albustic were excluded from study. Micral test, a immunological rapid dip stick semi-qualitative technique for detection of microalbuminuria was used for estimation of microalbuminuria. Analysis and conclusion were made with collected clinical data.

Group A - Type DM patients who were positive for microalbuminuria (Micral Positive) 50 patients. 
Group B - Type 2 DM patients who were negative for microalbuminuria (Micral Positive) 50 patients.

A detailed clinical analysis of these 100 patients was made and the differences and their implications are discussed.

\section{RESULTS AND DISCUSSION}

There is no significant difference between Age $(\mathrm{P}=0.816)$. The ages ranged from 32 to 78 yrs. and 32 to 78 yrs. in Micral positive and Micral negative groups respectively. These observations are similar to the studies done by Vishwanathan $\mathrm{M}^{4}$ et al (1992) in Madras and Ritz E5 et al (1994) in Europe.

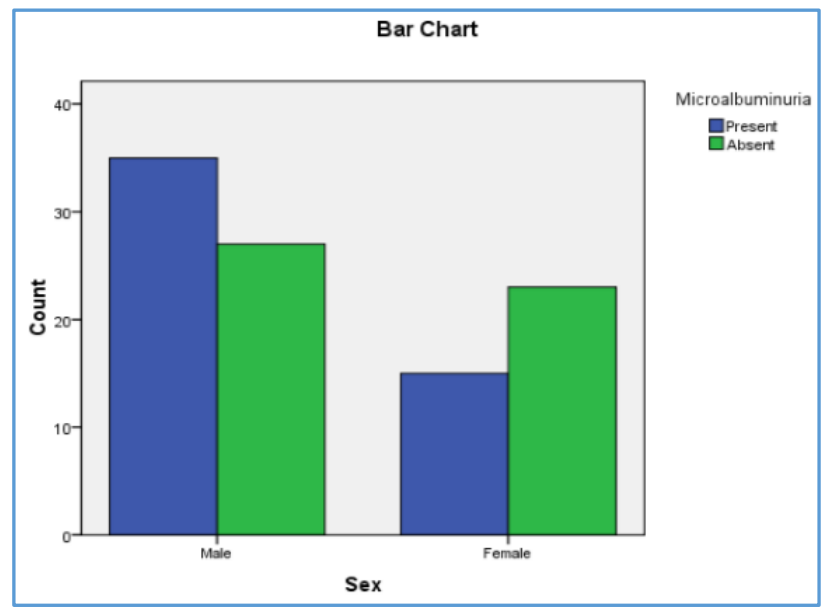

Graph 1

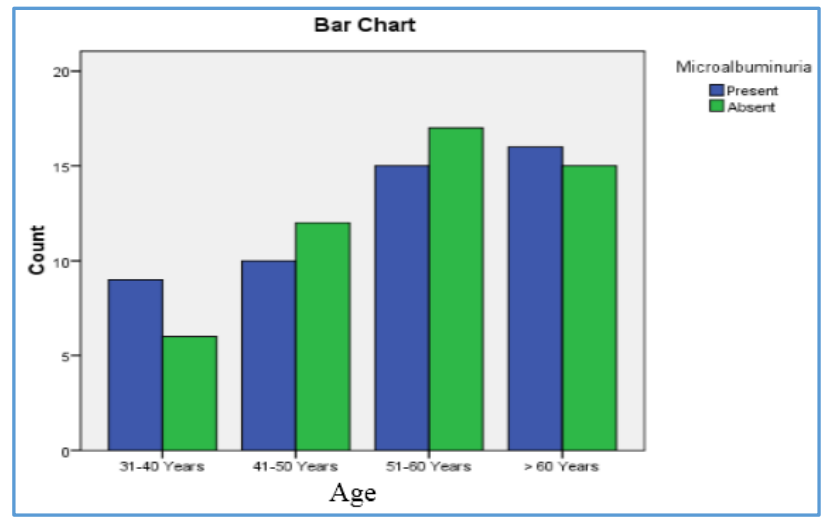

Graph 2

Data presented in Table 1 shows that poor glycaemic control $(\mathrm{P}=<0.001)$ and $66 \%$ in Micra positive group, $16 \%$ in Micral negative group.

Smoking ( $\mathrm{P}=0.016)$ observed in $32 \%$ of Micral positive patients and $12 \%$ of Micral negative patients.

Family history of diabetes $(\mathrm{P}=0.585)$ and $18 \%$ in the Micral positive group, $14 \%$ in the Micral negative group.

\begin{tabular}{|c|c|c|c|}
\hline & $\begin{array}{c}\text { Micral } \\
\text { Positive }\end{array}$ & $\begin{array}{c}\text { Micral } \\
\text { Negative }\end{array}$ & $\begin{array}{c}\text { "P" } \\
\text { Value }\end{array}$ \\
\hline Poor Glycaemic Control & $66 \%$ & $16 \%$ & $<0.001$ \\
\hline Smoking & $32 \%$ & $12 \%$ & 0.016 \\
\hline $\begin{array}{c}\text { Family History of } \\
\text { Diabetes Mellitus }\end{array}$ & $18 \%$ & $14 \%$ & 0.585 \\
\hline $\begin{array}{c}\text { Family History of } \\
\text { Nephropathy }\end{array}$ & $4 \%$ & $0 \%$ & 0.153 \\
\hline \multicolumn{3}{|c}{ Table 1 } \\
\hline
\end{tabular}

\begin{tabular}{|c|c|c|c|}
\hline & \multicolumn{3}{|c|}{ Microalbuminuria } \\
\hline HBA1C & Present & Absent & Total \\
\hline$<7$ & 17 & 41 & 58 \\
\hline$>7$ & 33 & 9 & 42 \\
\hline Total & 50 & 50 & 100 \\
\hline \multicolumn{2}{|r|}{ Table 2. HbA1c and Microalbuminuria } \\
\hline
\end{tabular}

$\mathrm{X}^{2}=21.71, \mathrm{P}<0.001, \mathrm{CI}=95 \%$.

Data presented in Table 2 shows that poor glycaemic control $(\mathrm{P}=<0.001)$ and $66 \%$ in Micra positive group, $16 \%$ in Micral negative group. The present study reveals a strong association of microalbuminuria with poor glycaemic control. Strong association of poor glycaemic control to microalbuminuria in type 2 DM was shown in studies by Mogensen C E et al. ${ }^{6}$ The DCCT study also emphasises the role of glycaemic control in the prevention and control of microvascular complications in type 1 DM patients. Hence, strict glycaemic control will be an important intervention in arresting the progression of early nephropathy.

\begin{tabular}{|c|c|c|c|}
\hline & \multicolumn{3}{|c|}{ Microalbuminuria } \\
\hline Smoking & Present & Absent & Total \\
\hline Present & 16 & 6 & 22 \\
\hline Absent & 34 & 44 & 78 \\
\hline Total & $\mathbf{5 0}$ & $\mathbf{5 0}$ & $\mathbf{1 0 0}$ \\
\hline Table 3. Habit of Smoking and Microalbuminuria \\
\hline
\end{tabular}

$\mathrm{X}^{2}=4.72, \mathrm{P}<0.016, \mathrm{CI}=95 \%$.

Smoking $(\mathrm{P}=0.016)$ observed in $32 \%$ of Micral positive patients, $12 \%$ of Micral negative patients. Till now there are no studies to demonstrate a direct relationship of smoking to microalbuminuria. However, as both smoking and microalbuminuria are risk factors and risk predictors of vascular disease respectively, it is advisable to stop smoking This could also be one of the interventions in arresting the progression of diabetic nephropathy.

\begin{tabular}{|c|c|c|c|}
\hline & \multicolumn{3}{|c|}{ Microalbuminuria } \\
\hline Hypertension & Present & Absent & Total \\
\hline Present & 27 & 7 & 34 \\
\hline Absent & 23 & 43 & 66 \\
\hline Total & $\mathbf{5 0}$ & $\mathbf{5 0}$ & $\mathbf{1 0 0}$ \\
\hline \multicolumn{3}{|c|}{ Table 4. Hypertension and Microalbuminuria }
\end{tabular}

$\mathrm{X}^{2}=16.08, \mathrm{P}<0.001, \mathrm{CI}=95 \%$.

This study showed higher prevalence of hypertension (54\%) in Micral positive group compared to $14 \%$ in Micral negative group. Studies by Mogensen C E et al. ${ }^{6}$ Most of these patients developed hypertension after a significant time gap after detection of diabetes. The mean duration of hypertension in Micral positive group was years compared to mean duration of diabetes of years.

\begin{tabular}{|c|c|c|c|}
\hline & \multicolumn{3}{|c|}{ Microalbuminuria } \\
\hline Retinopathy & Present & Absent & Total \\
\hline Present & 22 & 4 & 26 \\
\hline Absent & 28 & 46 & 74 \\
\hline Total & $\mathbf{5 0}$ & $\mathbf{5 0}$ & $\mathbf{1 0 0}$ \\
\hline Table 5. Retinopathy and Microalbuminuria \\
\hline
\end{tabular}

$\mathrm{X}^{2}=15.02, \mathrm{P}<0.001, \mathrm{CI}=95 \%$. 
Retinopathy was seen in $44 \%$ of Micral positive cases and $8 \%$ of Micral negative type $2 \mathrm{DM}$. In a study by Ravid et al ${ }^{7}$ in normotensive type 2 DM with microalbuminuria the prevalence of retinopathy was $26 \%$, while studies by Eggersten et al ${ }^{8}$ revealed a prevalence of $40 \%$. This observation stresses the need for careful evidence of retinopathy in all cases of microalbuminuria and vice versa.

The presence of diabetic retinopathy serves as a marker for diabetic nephropathy, helps in distinguishing diabetic from non-diabetic nephropathy.

\begin{tabular}{|c|c|c|c|}
\hline & \multicolumn{3}{|c|}{ Microalbuminuria } \\
\hline Ischaemic Heart Disease & Present & Absent & Total \\
\hline Present & 14 & 3 & 17 \\
\hline Absent & 36 & 47 & 83 \\
\hline Total & $\mathbf{5 0}$ & $\mathbf{5 0}$ & $\mathbf{1 0 0}$ \\
\hline Table 6. Ischaemic Heart Disease and Microalbuminuria \\
\hline
\end{tabular}

$\mathrm{X}^{2}=7.08, \mathrm{P}<0.007, \mathrm{CI}=95 \%$.

The prevalence of ischaemic heart disease was also higher in Micral positive group (28\%) compared to Micral negative group (6\%). This observation is similar to the higher prevalence of ischaemic heart disease seen in European patients by Mattock et $\mathrm{al}^{9}(1992)$ in U.K. and Mogensen et al 6 (1992). This observation supports the view that microalbuminuria is not only an indicator of early nephropathy, but also an indicator of diffuse vascular disease and a predictor of cardiovascular deaths.

\begin{tabular}{|c|c|c|c|}
\hline & \multicolumn{3}{|c|}{ Microalbuminuria } \\
\hline Nephropathy & Present & Absent & Total \\
\hline Present & 14 & 03 & 17 \\
\hline Absent & 36 & 47 & 83 \\
\hline Total & $\mathbf{5 0}$ & $\mathbf{5 0}$ & $\mathbf{1 0 0}$ \\
\hline \multicolumn{2}{|c}{ Table 7. Neuropathy and Microalbuminuria } \\
\hline
\end{tabular}

$\mathrm{X}^{2}=7.08, \mathrm{P}<0.007, \mathrm{CI}=95 \%$.

Neuropathy is also a major microvascular complication. In our study, neuropathy predominantly sensory and peripheral was found in $28 \%$ of Micral positive patients. In a study by Dyck et al,10 Mayo Clinic 59\% had some form of neuropathy in type $2 \mathrm{DM}$ patients with microalbuminuria. Autonomic neuropathy was present in $28 \%$ of the patients in our study compared to $5 \%$ in the study by Dyck et al.

\begin{tabular}{|c|c|c|c|}
\hline & \multicolumn{3}{|c|}{ Microalbuminuria } \\
\hline Total Cholesterol & Present & Absent & Total \\
\hline$<200$ & 12 & 31 & 43 \\
\hline$>200$ & 38 & 19 & 57 \\
\hline Total & 50 & 50 & 100 \\
\hline Table 8. Total Cholesterol and Microalbuminuria \\
\hline
\end{tabular}

$\mathrm{X}^{2}=13.21, \mathrm{P}<0.002, \mathrm{CI}=95 \%$.

Serum cholesterol, triglycerides and LDL cholesterol were measured in both the groups. There was a significant difference in these levels in both groups. $\mathrm{P}$ value $<0.001$. These results are similar to study done in MLB Medical College, Jhansi, Navneet Agarwal et al ${ }^{11}$ (March 2011). Eberhard Ritz et al did not find any significant differences in lipid levels of microalbuminuric type 2 patients compared to non-microalbuminuric type 2 patients.

\section{CONCLUSION}

- Age and Sex, do not seem to be directly related to the development of microalbuminuria in type 2 DM patients.

- $\quad$ Smoking has a strong association with the development of microalbuminuria. Hence, discontinuation of smoking will be an important intervention in arresting the progression of early nephropathy.

- $\quad$ Poor glycaemic control is also strongly associated with type $2 \mathrm{DM}$ patients with microalbuminuria.

- $\quad$ The DCCT study shows the importance of tight glycaemic control in the prevention of microvascular complications. So a good glycaemic control should be aimed at in patients with early nephropathy.

- Hypertension has a strong association with the development of microalbuminuria.

- Ischaemic Heart Disease is also common in type 2 DM with microalbuminuria.

- Retinopathy has a very strong association with microalbuminuria.

- Neuropathy another microvascular complication is also strongly associated with microalbuminuria.

- Serum cholesterol, triglycerides and LDL levels were significantly higher in microalbuminuric patients.

- Our study brings the importance of detection of microalbuminuria. Such early detection will help in arresting the further progression of nephropathy and prevent development of end-stage renal disease, which is a major cause of morbidity and mortality in type 2 patients. While control of DM, screening for microalbuminuria should be performed at least once a year.

\section{REFERENCES}

[1] Longo D, Fauci A, Kasper D, et al. Harrison's principles of internal medicine. 18 $8^{\text {th }}$ edn. Chapter 344, McGraw Hill Professional 2011.

[2] Chugh KS. Three decades of nephrology. J Postgrad 1994;40(3):103-8.

[3] Mani MK. Prevention of chronic renal failure at the community level. Kidney Int 2003;83:S86-9.

[4] Viswanathan M, McCarthy MI, Snehalatha C, et al. Familial aggregation of type 2 (non-insulindependent) diabetes mellitus in south India; absence of excess maternal transmission. Diabet Med 1996;13(3):232-7.

[5] Ritz E, Keller CK, Bergis KH, et al. Renal involvement in type II diabetes. Current Opinion in Nephrology and Hypertension 1994;3(2):137-44.

[6] Mogensen CE. Microalbuminuria predicts clinical proteinuria and early mortality in maturity-onset diabetes. The New England journal of medicine 1984;310(6):356-60.

[7] Ravid M, Savin H, Jutrin I, et al. Long-term stabilizing effect of angiotensin-converting enzyme inhibition on plasma creatinine and on proteinuria in normotensive type II diabetic patients. Annals of internal medicine 1993;118(8):577.

[8] Eggertsen R, Kalm H, Blohmé G. The value of screening for retinopathy and microalbuminuria in patients with type 2 diabetes in primary health care. Scandinavian journal of primary health care 1993;11(2):135-40. 


\section{Jemds.com}

[9] Mattock MB, Morrish NJ, Viberti G, et al. Prospective study of microalbuminuria as predictor of mortality in NIDDM. Diabetes 1992;41(6):736-41.

[10] Dyck PJ, Kratz KM, Karnes JL, et al. The prevalence by staged severity of various types of diabetic neuropathy, retinopathy, and nephropathy in a population-based cohort: the Rochester diabetic neuropathy study. Neurology 1993;43(4):817-24.
Original Research Article

[11] Agarwal N, Sengar NS, Jain PK, et al. Nephropathy in newly diagnosed type 2 diabetics with special stress on the role of Hypertension. JAPI 2011;59:145-7. 